\title{
Efecto de un programa de entrenamiento interválico aeróbico de alta intensidad en población escolar femenina con sobrepeso u obesidad Effect of a high intensity aerobic interval training program on an overweight or obese female school population \\ Albeiro Antonio Dávila Grisalez, César Augusto Mazuera Quiceno, Anggy Lorena Carreño Herrera, José Leandro Henao Corrales \\ Universidad Unidad Central del Valle del Cauca (Colombia)
}

\begin{abstract}
Resumen. El presente estudio de alcance explicativo, realizado bajo un diseño cuasiexperimental de preprueba-posprueba y grupo control, determinó el efecto de un programa de entrenamiento interválico aeróbico de alta intensidad (HIIT) que utilizó juegos motores y circuitos sociomotrices, durante 16 semanas (tres sesiones semanales) para el incremento del consumo de oxígeno pico $\left(\mathrm{VO}_{2 \text { pico }}\right)$ y disminución del índice de masa corporal (IMC) y porcentaje de grasa (\%GC) en niñas con sobrepeso u obesidad de 10 y 11 años de la sede Marino Dávalos de la Institución Educativa Moderna de Tuluá, distribuidas en dos grupos, experimental $(\mathrm{n}=8)$ y control ( $\mathrm{n}=8)$. Entre los resultados más importantes, se destaca el aumento del $\mathrm{VO}_{\text {2pico }}$ en $3.6 \mathrm{ml} / \mathrm{kg} / \mathrm{min}$, la disminución del IMC en 1.62 y del \%GC donde se apreció una disminución del $3.72 \%$ en el grupo experimental; mientras que en el grupo control, no se observaron modificaciones estadísticamente significativas; concluyendo el estudio que el programa HIIT que utilizó como medio los juegos motores y circuitos sociomotrices, presentó efectos significativos en el incremento del $\mathrm{VO}_{2 \text { ico }}$ y disminución del IMC y el \%GC de las niñas con sobrepeso u obesidad, validando de esta manera la hipótesis de investigación, con niveles de significancia estadística $(p<.05)$ para la prueba de muestras relacionadas ( $p=.000$ para el $\mathrm{VO}_{2 \text { pico }}, p=.000$ para $\% \mathrm{GC}$ y $p=.000$ para IMC).
\end{abstract}

Palabras clave: Entrenamiento interválico aeróbico de alta intensidad, juegos motores, niñas, circuitos sociomotrices, obesidad, sobrepeso.

Abstract. The present explanatory scope study, carried out under a quasi-experimental design of pretest-posttest and control group, determined the effect of a high intensity aerobic interval training (HIIT) program that used motor games and sociomotor circuits, for 16 weeks (three sessions weekly) for the increase in peak oxygen consumption $\left(\mathrm{VO}_{2 \text { peak }}\right)$ and decrease in body mass index (BMI) and percentage of fat $(\% \mathrm{GC})$ in overweight or obese girls of 10 and 11 years of age from the Marino Dávalos headquarters of the Institution Educativa Moderna de Tuluá, divided into two groups, experimental $(n=8)$ and control $(n=8)$. Among the most important results, the increase in $\mathrm{VO}_{2 \text { peak }}$ by $3.6 \mathrm{ml} / \mathrm{kg} / \mathrm{min}$, the decrease in $\mathrm{BMI}$ by 1.62 and the $\% \mathrm{GC}$ where a decrease of $3.72 \%$ was observed in the experimental group stand out; while in the control group, no statistically significant changes were observed; The study concluded that the HIIT program that used motor games and sociomotor circuits as a means, presented significant effects in the increase of the $\mathrm{VO}_{2 \text { peak }}$ and decrease of the $\mathrm{BMI}$ and the $\% \mathrm{GC}$ of the girls with overweight or obesity, thus validating the research hypothesis. , with levels of statistical significance $(p<.05)$ for the test of related samples $\left(p=.000\right.$ for $\mathrm{VO}_{2 \text { peak }}, p=.000$ for $\% \mathrm{GC}$ and $p=.000$ for $\left.\mathrm{BMI}\right)$.

Keywords: High intensity aerobic interval training, motor games, girls, socio-motor circuits, obesity, overweight.

\section{Introducción}

La obesidad infantil es una de las problemáticas de salud pública más graves del siglo XXI, ya que en los últimos 40 años el número de niños obesos entre 5 y 19 años creció más de 10 veces, es decir, de 11 millones de niños obesos que existían en los años 70, se incrementó a 124 millones en el 2016 (Imperial College de Londres \& OMS, 2017). Ahora bien, a nivel de Latinoamérica y el Caribe se ha evidenciado un incremento del sobrepeso en poblaciones infantiles del 5.1\% al $7 \%$ entre 1990 y el 2015 , en cuanto a la zona del Caribe el $6.8 \%$ de los niños presentaron sobrepeso, mientras que, en Sudamérica fue del 7.4\% (FAO \& OPS, 2017). En un estudio actual, se encontró que el $25 \%$ de la población escolar de Centroamérica presentó sobrepeso y obesidad (Álvarez, Herrera, Herrera, Villalobos, \& Araya, 2020). Con respecto al sobrepeso y la obesidad en Colombia, se observó un incremento del $18.8 \%$ al $24.4 \%$ entre el 2010 y el 2015 (Ministerio de Salud y Protección Social, 2017). A nivel local, en la sede Marino Dávalos de la Institución Educativa Moderna de Tuluá, se encontró que el $21 \%$ de la población infantil de los grados tercero, cuarto y quinto presentaron sobrepeso $\mathrm{u}$ obesidad.

Fecha recepción: 03-02-20. Fecha de aceptación: 03-08-20 César Augusto Mazuera Quiceno cmazuera@uceva.edu.co
Tradicionalmente se ha prescripto ejercicio cardiovascular utilizando métodos continuos uniformes de intensidad moderada para el control del sobrepeso u obesidad, recurriendo a ejercicios de carácter cíclico, como la carrera pedestre, montar en bicicleta, practicar natación al aire libre o ejercicio cíclicos en centro de acondicionamiento como la práctica en banda trotadora, elíptica, spinning; pero tanto la metodología de carácter continuo como la utilización de medios de carácter cíclicos resultan muy monótonos y aburridos para los niños (Hassink, 2010), lo que dificulta la adherencia a la práctica de actividad física sistemática. Al respecto, son diversos los estudios que han utilizado actividades cíclicas, tal es el caso de las investigaciones de Viñuela, Vera, Colomer, Márquez y Romero (2016); Heydari, Freund y Boutcher (2012); Galdames-Maliqueo, Huerta-Ojeda, Chirosa-Ríos, Cáceres-Serrano y Reyes-Amigo (2017); Camacho et al. (2016); Vargas-Ortiz, Macías-Cervantes, DíazCisnero y Pérez-Vázquez (2014). De allí que, en el presente estudio se haya planteado como hipótesis de investigación que un programa de entrenamiento interválico aeróbico de alta intensidad (HIIT) que utiliza juegos motores y circuitos sociomotrices, presenta efectos significativos en la disminución del índice de masa corporal (IMC) y porcentaje de grasa (\%GC), y en el aumento del consumo de oxígeno pico $\left(\mathrm{VO}_{2 \text { ico }}\right)$ de un grupo de niñas con sobrepeso u obesidad.

El HIIT, es un método de entrenamiento de la resistencia 
que se caracteriza por utilizar series breves e intermitentes a alta intensidad, intercaladas con períodos de recuperación con ejercicios de baja intensidad (Gibala, Little, MacDonald, \& Hawley, 2012). De igual forma, Laursen y Buchheit (2019) consideran el HIIT como secuencias de esfuerzo de alta intensidad, ejecutadas por encima del umbral de lactato alternado con secuencias de ejercicio de moderada intensidad o de recuperación pasiva, proponiendo una clasificación de acuerdo a la duración e intensidad del estímulo (HIIT de larga duración, HIIT de corta duración, HIIT de entrenamiento repetido de sprint (RST) y el entrenamiento interválico de sprint (SIT)). Así mismo, Bond et al. (2015) consideran esta metodología como apropiada para mejorar la composición corporal y la salud metabólica, Boutcher (2011) explica que su eficacia se fundamenta en la capacidad para reducir el tejido graso a nivel subcutáneo y abdominal, al compararse con otras metodologías de intervención.

Son varias las razones que hacen relevante el uso del método HIIT en poblaciones infantiles. La más crucial, está relacionada con las características interválicas del HIIT que se identifican con los patrones motores de actividad diaria cambiantes de los niños, al alternar esfuerzos cortos de alta intensidad con secuencias de baja y moderada exigencia, como se pudo evidenciar en un estudio observacional (Bailey, et al., 1995); además, se ha demostrado que la práctica de ejercicio físico en zona fat max intercalada con esfuerzos cortos de alta intensidad (4 segundos) cada 2 minutos durante 30 minutos en esta zona de entrenamiento, incrementó el gasto energético sin interrumpir la oxidación de grasas, pero aún más importante, fue más placentero según la percepción de niños con sobrepeso, pudiendo ser un factor de gran influencia a la adherencia al ejercicio físico a largo plazo (Crisp, Fournier, Licari, Braham, \& Guelfi, 2012); de la misma forma, la velocidad de recuperación de la frecuencia cardiaca y consumo de oxígeno encontrada en los niños fue mucho más rápida que en jóvenes o adultos al realizar esfuerzos de 30 segundos de alta intensidad (Hebestreit, Mimuray, \& BarOr, 1993); por último, es importante utilizar el método HIIT, pero tratando de implementar en lo posible medios de entrenamiento que se puedan utilizar en las sesiones de educación física y que además, presenten un alto componente lúdico y sociomotriz, para crear una mayor motivación de los participantes en los procesos investigativos con poblaciones escolares con sobrepeso u obesidad, siendo para este estudio la razón más importante.

Es por ello, que el objetivo de esta investigación consistió en determinar la incidencia de un programa de HIIT que utiliza juegos motores y circuitos sociomotrices en el IMC, $\% \mathrm{GC}$ y $\mathrm{VO}_{\text {2pico }}$ de un grupo de niñas con sobrepeso u obesidad.

\section{Materiales y métodos}

\section{Participantes}

El estudio presentó un alcance explicativo y un diseño cuasiexperimental de preprueba-posprueba y grupo control. En el caso del grupo experimental $(n=8)$ conformado por niñas con un rango de edad entre 10 y 11 años, peso corporal $M=43.9 \pm 5.1 \mathrm{~kg}$; talla $M=140.6 \pm 5.1 \mathrm{~cm} ; \% \mathrm{GC}$ de $M=41.4 \pm$ .94 y un IMC $M=22.1 \pm 1.7$. Ahora bien, el grupo control $(n=8)$ de igual forma estuvo conformado por niñas con un rango de edad entre 10 y 11 años, peso corporal $M=44.9 \pm$ $7.3 \mathrm{~kg}$; talla $M=141.3 \pm 6.5 \mathrm{~cm} ; \% \mathrm{GC}$ de $M=40.5 \pm 2.45 \mathrm{y}$ un IMC $M=22.8 \pm 2.81$.

Las niñas que pertenecían a la Institución Educativa Moderna de Tuluá, sede Marino Dávalos, participaron de forma voluntaria y adicionalmente, los padres, docentes y niñas involucradas en la investigación fueron informados acerca de los riegos y beneficios del estudio. Por lo tanto, los padres de familia firmaron un consentimiento informado antes de comenzar las mediciones y la intervención, siguiendo la declaración de Helsinki. Como criterios de inclusión las niñas debían presentar obesidad o sobrepeso, tener entre 10 y 11 años, y no pertenecer a escuelas de formación deportiva o que realizaran actividad física sistemática extracurricular.

\section{Instrumentos de evaluación}

Mediciones antropométricas. La talla $(\mathrm{cm})$ se evaluó utilizando un estadiómetro marca Bodymeter 206, con un nivel de precisión de $1 \mathrm{~mm}$. La valoración del peso corporal, fue determino mediante la balanza de piso profesional Health o Meter. En el caso de la medición del porcentaje graso se utilizó un calibrador Harpenden Skinfold, con un rango de medición de 0-80 mm, una graduación de 0-20 mm y una precisión de $0.2 \mathrm{~mm}$, ejerciendo una presión entre yunques de $10 \mathrm{~g} / \mathrm{m}^{2}$. Por último, para valorar el IMC, se utilizó la ecuación de relación peso $(\mathrm{kg})$ sobre talla en metros al cuadrado $\left(\mathrm{kg} / \mathrm{m}^{2}\right)$ y la clasificación antropométrica del estado nutricional para población infantil y adolescente, de damas y varones, de 5 a 17 años, y gráficos de los patrones de crecimiento establecidos por la Organización Mundial de la Salud (OMS) según indicador, y punto de corte establecido en el cuadro 3 de la resolución 2465 de 2016 (Ministerio de Salud y Protección Social, 2016).

Medición componente cardiorrespiratorios. Para la valoración del $\mathrm{VO}_{2 \text { pico }}$, se utilizó el test de Course Navette y se hizo uso de la ecuación específica para poblaciones de 8 a 19 años: $31.025+(3.238 x$ Vf $)-(3.248 x$ e $)+(0.1536 x$ Vfxe $)$ (Alba, 2010), donde Vf indica la velocidad alcanzada en en la última etapa del test, mientras que la e representa la edad del evaluado.

\section{Procedimiento de evaluación}

Las evaluaciones se realizaron en horas de la mañana en el horario curricular, 3 horas después de haber desayunado un menú ligero que aportara principalmente carbohidratos complejos, llevándose a cabo en un salón climatizado de la Institución Educativa, a una temperatura promedio de $26^{\circ} \mathrm{C}$. En primera instancia, se realizaron las valoraciones antropométricas iniciando con la medición de la talla de las niñas, para ello, se ubicaron junto a la pared sin zapatos haciendo contacto con talones y glúteos, asumiendo el plano de Frankfort. Seguidamente, se pesaron ubicándose sobre una báscula, portando solo licra y camisa corta, en el caso del porcentaje de grasa se utilizó el método doblemente indirecto a partir de los pliegues cutáneos específicos para población infantil (tricipital y pantorrilla) tomados en el lado derecho del cuerpo, para la determinación del \%GC se recurrió a la ecuación de Lohman (como se cita en Katch, McArdle, $\&$ Katch, 2014). Además, para valorar IMC se utilizó la ecua- 
ción de relación peso $(\mathrm{kg})$ sobre talla en metros al cuadrado $\left(\mathrm{kg} / \mathrm{m}^{2}\right)$ y el resultado se relacionó con la edad en añosmeses(IMC/E), dicho resultado se valoró según indicador y punto de corte establecido en el cuadro 3 de la resolución 2465 de 2016 (Ministerio de Salud y Protección Social, 2016), clasificándose con sobrepeso los valores encontrados en las niñas que se ubicaron entre las líneas de puntuación mayor a 1 , y menor o igual a 2 desviaciones estándar, mientras que se consideraron las niñas con obesidad, a aquellas que presentaron valores mayores a 2 desviaciones estándar.

Ahora bien para valorar el $\mathrm{VO}_{2 \text { pico }}$ se utilizó el test de Course Navette, en el cual las niñas recorrieron una distancia de 20 metros entre dos líneas demarcadas de forma paralela, teniendo que aumentar de forma progresiva la velocidad de desplazamiento exigida, con base en un audio pregrabado que informó cuando debían partir o incrementar la velocidad. Cada etapa dura 1 minuto, siendo $8,5 \mathrm{~km} / \mathrm{h}$ la velocidad inicial, la cual va aumentando $0,5 \mathrm{~km} / \mathrm{h}$ por fase y se da por terminado el test cuando la niña no alcanza a llegar a la línea al sonar el audio en tres ocasiones.

\section{Intervención}

El programa de intervención fue estructurado en cuatro fases, cada una respondiendo a la utilización de los diferentes tipos de HIIT, tomando como referencia la clasificación propuesta por Buchheit y Laursen (2013). Como se puede identificar en el plan gráfico descrito en la figura 1, llevado a cabo por el grupo experimental el cual realizó tres sesiones semanales desarrolladas en el horario curricular dedicado a la asignatura de educación física. Por otra parte, las otras ocho niñas solo fueron evaluadas al inicio y final del proceso investigativo, continuando con sus tres sesiones habituales de educación física en la Institución Educativa, conformando el grupo control de este estudio.

Para el control de la intensidad de esfuerzo, se parte de la clasificación de los tipos de HIIT propuesta por Buchheit y Laursen (2013), en este caso, la duración de los esfuerzos, la velocidad de ejecución y la complejidad de los circuitos o juegos motores determinaron el nivel de esfuerzo realizado por las niñas. Los circuitos o juegos motores planteados presentaron diferentes tipos de duración de acuerdo a cada fase. En la primera fase dichos juegos motores presentaron una secuencia de duración de 2 a 4 minutos, en la segunda fase la duración de estos juegos fue de 1 a 2 minutos, en la tercer fase se realizaron juegos cuya duración oscilaba entre 30 y 45 segundos, por último, en la cuarta fase la secuencia de esfuerzos de circuitos o juegos motores duraba entre 10 a 30 segundos y de 3 a 7 segundos.

Es importante resaltar que, el plantear medios de entrenamiento como el juego motor o circuitos, que denotan la ejecución de trabajos interválicos permiten la alternancia de secuencias de esfuerzo activas con pausas de recuperación incompletas, imitando de esta forma las características del HIIT, que como señalan López y Vicente (2018) a partir del juego los niños construyen de forma espontánea trabajos interválicos. Cabe anotar, que al realizarse estas actividades en espacios abiertos, es más difícil el control de la intensidad a partir de la frecuencia cardiaca o la percepción subjetiva, por lo que se recurrió a la velocidad de ejecución de los juegos o circuitos y al nivel de complejidad de las tareas planteadas, en las que como se puede apreciar en la figura 1 , se utilizaron niveles de interacción de cooperación, de oposición o de cooperación-oposición, lo que implica una mayor motivación y exigencia emocional que suscita niveles de frecuencia cardiaca altos al tener que resolver situaciones de juego de forma colectiva.

Un aspecto relevante en el proceso de intervención fue el desarrollo de la competencia axiológica corporal impartida a las niñas y padres de familia de ambos grupos una vez a la semana, enfatizando en los hábitos nutricionales, estilos de vida saludables y normas de convivencia en grupo, con lo que se aclara que en este estudio no se plantearon dietas o menús estrictos para las niñas, con el objetivo de darle mayor importancia al diseño de estrategias pedagógicas a partir del movimiento y del desarrollo de la competencia motriz y axiológica corporal, especificas a la educación física recreación y deporte.

En la primera fase, descrita en la figura 1, se realizaron circuitos y juegos motores, utilizando HIIT de larga duración, tres sesiones semanales en las que la duración de las secuencias de esfuerzo de los juegos o circuitos motores oscilaron entre 3 y 4 minutos, con intervalos de recuperación

\begin{tabular}{|c|c|c|c|c|c|c|c|c|c|c|c|c|c|c|c|c|}
\hline \multirow{2}{*}{\begin{tabular}{|l|} 
Fase \\
Mes \\
\end{tabular}} & \multicolumn{4}{|c|}{ HIIT larga duración } & \multicolumn{4}{|c|}{ HIIT media duración } & \multicolumn{4}{|c|}{ HIIT corta duración } & \multicolumn{4}{|c|}{ HIIT sprints } \\
\hline & \multirow{2}{*}{\begin{tabular}{|l} 
Enero \\
1
\end{tabular}} & \multicolumn{3}{|c|}{ Febrero } & & \multicolumn{4}{|c|}{ Marzo } & \multicolumn{4}{|c|}{ Abril } & \multicolumn{3}{|c|}{ Mayo } \\
\hline & & 2 & 3 & 4 & 5 & 6 & 7 & 8 & 9 & 10 & 11 & 12 & 13 & 14 & 15 & 16 \\
\hline Fecha de iniciación y finalización & $28-2 / 02$ & $4-9 / 02$ & $11-16 / 02$ & $18-22 / 02$ & $25-2 / 03$ & $4-9 / 03$ & $\mid 11-16 / 03$ & $18-23 / 03$ & $25-30 / 03$ & $1-6 / 04$ & $8-13 / 04$ & \begin{tabular}{|l}
$15-20 / 04$ \\
\end{tabular} & $22-27 / 04$ & $29-4 / 05$ & $6-10 / 05$ & $13-17 / 05$ \\
\hline Sesiones por semana & 3 & 3 & 3 & 3 & 3 & 3 & 3 & 3 & 3 & 3 & \begin{tabular}{|c|}
3 \\
\end{tabular} & 3 & 3 & 3 & 3 & 2 \\
\hline Volumen promedio sesión ( $\mathrm{min}$ ) & $60^{\prime}$ & 60 ' & $60^{\prime}$ & $60^{\prime}$ & 70 ' & $70^{\prime}$ & $70^{\prime}$ & $70^{\prime}$ & $80^{\prime}$ & $80^{\prime}$ & $80^{\prime}$ & $80^{\prime}$ & $80^{\prime}$ & $80^{\prime}$ & $80^{\prime}$ & $120^{\prime}$ \\
\hline Volumen total semana (min) & $180^{\prime}$ & $180^{\prime}$ & $180^{\prime}$ & $180^{\prime}$ & $210^{\prime}$ & $210^{\prime}$ & $210^{\prime}$ & $210^{\prime}$ & $240^{\prime}$ & $240^{\prime}$ & $240^{\prime}$ & $240^{\prime}$ & $240^{\prime}$ & $240^{\prime}$ & 240 ' & 240 ' \\
\hline Calentamiento y vuelta a la calma & 30 ' & 30 ' & $30^{\prime}$ & $30^{\prime}$ & $30^{\prime}$ & $30^{\prime}$ & $30^{\prime}$ & $30^{\prime}$ & 45 ' & $45^{\prime}$ & $45^{\prime}$ & 45 ' & $45^{\prime}$ & $45^{\prime}$ & 45 ' & \\
\hline \begin{tabular}{|l} 
Actividades Fortalecimiento competencias axiológicas corporal \\
\end{tabular} & $30^{\prime}$ & 30 ' & 30 ' & $30^{\prime}$ & $30^{\prime}$ & 30 ' & $30^{\prime}$ & $30^{\prime}$ & $30^{\prime}$ & $30^{\prime}$ & 30 ' & $30^{\prime}$ & $30^{\prime}$ & $30^{\prime}$ & $30^{\prime}$ & \\
\hline Juegos motores HIIT largos $\left(2^{\prime}-4^{\prime}\right)$ & $90^{\prime}$ & $90^{\prime}$ & 75 ' & 75 ' & 75 ' & 45 ' & $45^{\prime}$ & 15 ' & & & & & & & & \\
\hline Circuitos motores de coordiación motriz y HMB locomotrices y manipulación & 45 & 45 , & 15 , & 15 ' & 15 ' & 15 ' & 15 , & & & & & & & & & \\
\hline Juegos motores de oposición & $30^{\prime}$ & 30 ' & 30 ' & 30 ' & 30 ' & 15 ' & 15 , & & & & & & & & & \\
\hline Circuitos o juegos motores de cooperación - oposición & 15 ' & 15 ' & 30 ' & 30 ' & 30 ' & 15 ' & 15 ' & 15 ' & & & & & & & & \\
\hline \begin{tabular}{|l|} 
Juegos motores HIIT medios $\left(1^{\prime}-2^{\prime}\right)$ \\
\end{tabular} & $30^{\prime}$ & 30 ' & 45 , & 45 , & 75 , & 105 & 90 ' & 105 ' & $60^{\prime}$ & 45 ' & 30 ' & $30^{\prime}$ & & & & \\
\hline Circuitos motores de coordiación motriz y HMB locomotrices y manipulación & 15 ' & 15 ' & 30 ' & $30^{\prime}$ & 30 ' & 45 , & $30^{\prime}$ & 30 ' & & & & & & & & \\
\hline Juegos motores de oposición & & & & & & 30 ' & $30^{\prime}$ & $30^{\prime}$ & $30^{\prime}$ & 15 ' & 15 ' & 15 ' & & & & \\
\hline Circuitos o juegos motores de cooperación - oposición & 15 ' & 15 ' & 15 ' & 15 ' & 15 ' & $30^{\prime}$ & $30^{\prime}$ & 45, & $30^{\prime}$ & $30^{\prime}$ & 15 ' & 15 ' & & & & \\
\hline Juegos motores HIIT cortos $\left(31\right.$ " a $\left.<45^{\prime \prime}\right)$ & & & & & & & 15, & $30^{\prime}$ & 105 ' & $120^{\prime}$ & $105^{\prime}$ & 105 ' & $75^{\prime}$ & $45^{\prime}$ & $45^{\prime}$ & \\
\hline Circuitos o juegos motores de cooperación & & & & & & & 15 ' & 15 ' & 45 ' & $45^{\prime}$ & $45^{\prime}$ & 45 ' & 15 ' & & & \\
\hline \begin{tabular}{|l|} 
Juegos motores de oposición \\
\end{tabular} & & & & & & & & 15 , & $30^{\prime}$ & $45^{\prime}$ & $30^{\prime}$ & $30^{\prime}$ & $30^{\prime}$ & 15 ' & 15 ' & \\
\hline Circuitos o juegos motores de cooperación - oposición & & & & & & & & & 30 ' & $30^{\prime}$ & $30^{\prime}$ & $30^{\prime}$ & $30^{\prime}$ & $30^{\prime}$ & $30^{\prime}$ & \\
\hline Juegos motores de HIIT SIT (10"-30") & & & & & & & & & & & $30^{\prime}$ & $30^{\prime}$ & $60^{\prime}$ & $75^{\prime}$ & 75 ' & \\
\hline Circuitos motores de cooperación & & & & & & & & & & & & & 15 , & 15 & 15 ' & \\
\hline Juegos motores de oposición & & & & & & & & & & & 10 ' & 10 ' & 30 ' & $30^{\prime}$ & $30^{\prime}$ & \\
\hline Juegos motores de cooperación - oposición & & & & & & & & & & & $20^{\prime}$ & 20 ' & 15 , & $30^{\prime}$ & $30^{\prime}$ & \\
\hline Juegos motores HIIT RST (3"-7") & & & & & & & & & & & & & $30^{\prime}$ & 45, & 45 , & \\
\hline Circuitos motores de cooperación & & & & & & & & & & & & & 10 ' & 15 ' & 15 ' & \\
\hline Juegos motores de oposición & & & & & & & & & & & & & $10^{\prime}$ & 15 ' & 15 ' & \\
\hline Juegos o juegos motores de cooperación - oposición & & & & & & & & & & & & & $10^{\prime}$ & 15 ' & 15 ' & \\
\hline Evaluación final & & & & & & & & & & & & & & & & $240^{\prime}$ \\
\hline
\end{tabular}

Figura 1. Programa de entrenamiento interválico aeróbico de alta intensidad. 
de 2 a 3 minutos entre serie. Durante esta fase de la propuesta se enfatizó en el control y perfeccionamiento de las diferentes habilidades motrices básicas inmersas en los juegos o circuitos motores, especialmente, en habilidades motrices como transportar, arrastrar o cargar implementos, pelotas, balones medicinales, etc., promoviendo de esta forma la correcta ejecución de movimientos con posturas adecuadas que permitieran formar una base sólida de la condición física, además, de educar la ejecución del movimiento e integración sociomotriz de los participantes.

Con respecto a la segunda fase, el programa planteó circuitos o juegos motores utilizando los HIIT de media duración (realización de secuencias de esfuerzo que duran entre 1 y 2 minutos) con intervalos de descanso de 1 a 2 minutos entre serie.

Ahora bien, en la tercera fase, se utilizaron circuitos o juegos motores bajo la metodología de HIIT cortos (duración de las series entre 31 y 45 segundos) y circuitos o juegos motores de HIIT de muy corta duración (duración de las series entre 10 y 30 segundos), con intervalos de recuperación entre serie de 45 a 90 segundos para los HIIT cortos, y de 20 a 60 segundos de intervalo de descanso entre serie en los HIIT muy cortos.

Por último, en la cuarta fase, se realizaron circuitos o juegos motores utilizando el HIIT de muy corta duración (10 a 30 segundos duración de cada serie) y el HIIT de sprints (duración de 3 a 7 segundos por serie), con intervalos de recuperación de 15 a 35 segundos.

\section{Proceso estadístico}

Los resultados se describen en media y desviación estándar. Ahora bien, para realizar las pruebas de hipótesis para muestras paramétricas, se realizaron los supuestos de normalidad (Shapiro-Wilk) y la prueba de homogeneidad (Estadístico de Levene) como se observa en la tabla 1. Por consiguiente al cumplir con los supuestos para pruebas de hipótesis paramétricas $(p>.05)$, se realizaron la prueba de $t$ Student para muestras relacionadas e independientes, y se empleó un nivel de significancia $(p<.05)$. Cabe mencionar que se utilizó el paquete estadístico SPSS Statistics versión 24.0.

\section{Resultados}

En la tabla 2 se observa que el grupo experimental preTabla 1.

Pruebas de normalidad y homogeneidad de varianzas de las variables de estudio

\begin{tabular}{|c|c|c|c|c|c|c|c|c|}
\hline \multirow{3}{*}{ Variables } & \multicolumn{4}{|c|}{ Grupo de investigación } & \multicolumn{4}{|c|}{ Grupo control } \\
\hline & \multicolumn{2}{|c|}{ Shapiro-Wilk $(p)$} & \multicolumn{2}{|c|}{ Levene $(p)$} & \multicolumn{2}{|c|}{ Shapiro-Wilk $(p)$} & \multicolumn{2}{|c|}{ Levene $(p)$} \\
\hline & Inicial & Final & Inicial & Final & Inicial & Final & Inicial & Final \\
\hline IMC & .372 & .303 & .384 & .782 & .499 & .586 & .384 & .782 \\
\hline$\%$ Grasa corporal & .348 & .128 & .335 & .096 & .802 & .537 & .335 & .096 \\
\hline $\mathrm{VO}_{2}$ pico $(\mathrm{ml} / \mathrm{Kg} / \mathrm{min})$ & .660 & .556 & .978 & .310 & .516 & .644 & .978 & .310 \\
\hline \multicolumn{9}{|l|}{$\begin{array}{l}\text { Tabla } 2 . \\
\text { Estadísticos descr }\end{array}$} \\
\hline Variables & \multicolumn{2}{|c|}{ Pret \pm DS } & \multicolumn{2}{|c|}{ Post \pm DS } & $\begin{array}{r}\text { Muestras } \\
\text { relacionadas }\end{array}$ & \multicolumn{3}{|c|}{$\begin{array}{c}\text { Muestras } \\
\text { independientes }(p)\end{array}$} \\
\hline Peso corporal (Kg) & \multicolumn{2}{|c|}{$43.9 \pm 5.1$} & \multicolumn{2}{|c|}{$41.6 \pm 4.9$} & - & & \\
\hline Talla $(\mathrm{cm})$ & \multirow{2}{*}{\multicolumn{2}{|c|}{$\begin{array}{l}140.6 \pm 5.1 \\
22.1 \pm 1.7\end{array}$}} & \multicolumn{2}{|c|}{$142.1 \pm 5.1$} & - & \multicolumn{3}{|c|}{-} \\
\hline IMC & & & \multicolumn{2}{|c|}{$20.5 \pm 1.6$} & .000 & \multicolumn{3}{|c|}{.039} \\
\hline$\%$ Grasa corporal & \multicolumn{2}{|c|}{$41.4 \pm .9$} & \multirow{2}{*}{\multicolumn{2}{|c|}{$\begin{array}{l}37.6 \pm 1.5 \\
41.1 \pm 2.1\end{array}$}} & .000 & \multirow{2}{*}{\multicolumn{3}{|c|}{.032}} \\
\hline $\mathrm{VO}_{2}$ pico $(\mathrm{ml} / \mathrm{Kg} / \mathrm{min})$ & \multicolumn{2}{|c|}{$37.5 \pm 2.0$} & & & .000 & & & \\
\hline \multicolumn{9}{|c|}{$\begin{array}{l}\text { Tabla } 3 . \\
\text { Estadísticos descriptivos y cambios de las variables de grupo control. }\end{array}$} \\
\hline Variables & \multicolumn{2}{|c|}{ Pret $\pm \mathrm{DS}$} & \multicolumn{2}{|c|}{ Post \pm DS } & \multicolumn{2}{|c|}{$\begin{array}{c}\text { Muestras } \\
\text { relacionadas }(p)\end{array}$} & \multicolumn{2}{|c|}{$\begin{array}{c}\text { Muestras } \\
\text { independientes }(p)\end{array}$} \\
\hline Peso corporal $(\mathrm{Kg})$ & \multicolumn{2}{|c|}{$44.9 \pm 7.2$} & \multirow{2}{*}{\multicolumn{2}{|c|}{$45.9 \pm 7.4$}} & - & & \multirow{2}{*}{\multicolumn{2}{|c|}{-}} \\
\hline Talla $(\mathrm{cm})$ & \multirow{2}{*}{\multicolumn{2}{|c|}{$\begin{array}{l}141.3 \pm 6.5 \\
22.8 \pm 2.8\end{array}$}} & $142.8 \pm 6.5$ & & \multicolumn{2}{|l|}{ - } & \multirow{2}{*}{\multicolumn{2}{|c|}{.039}} \\
\hline IMC & & & 22.4 & \pm 1.7 & .112 & & & \\
\hline \% Grasa corporal & & $.5 \pm 2.4$ & 40.6 & \pm 3.1 & .783 & & .032 & \\
\hline $\mathrm{VO}_{2}$ pico $(\mathrm{ml} / \mathrm{Kg} / \mathrm{min})$ & & $4 \pm 1.9$ & 36.9 & \pm 1.4 & .158 & & .001 & \\
\hline
\end{tabular}

sentó una disminución del peso corporal, mientras que en la talla se presentó un incremento. Ahora en el caso del IMC, \% $\mathrm{GC}$ y $\mathrm{VO}_{2 \text { ico }}$ se evidenciaron diferencias estadísticamente significativas $(p<.05)$ en las pruebas de hipótesis para muestras relacionadas e independientes.

Los resultados del grupo control descritos en la tabla 3 evidencian un aumento en el peso corporal al igual que en su talla. En cuanto al IMC, \% GC y VO 2pico $_{0}$ no se apreciaron diferencias estadísticamente significativas $(p>.05)$ en las pruebas de hipótesis para muestras relacionadas, pero si en las pruebas de hipótesis para muestras independientes $(p<.05)$.

\section{Discusión}

Esta investigación tuvo como objetivo determinar el efecto de un programa de entrenamiento interválico aeróbico de alta intensidad en el IMC, \%GC y $\mathrm{VO}_{2 \text { pico }}$ de un grupo de ocho niñas con sobrepeso u obesidad, implementándose un programa de HIIT que utilizó juegos motores y circuitos sociomotrices. Entre los principales hallazgos, se destacan las diferencias estadísticamente significativas presentadas en los valores de IMC ( $p=.000$ para muestras relacionadas y $p=.039$ para muestras independientes $), \% \mathrm{GC}(p=.000$ para muestras relacionadas y $p=.032$ para muestras independientes) y $\mathrm{VO}_{2 \text { pico }}(p=.000$ para muestras relacionadas y $p=.001$ para muestras independientes) de la población infantil con sobrepeso u obesidad intervenida en este estudio, lo que confirma la hipótesis planteada en esta investigación, evidenciando que el programa propuesto es una estrategia pedagógica eficaz y apropiada para la población infantil con sobrepeso u obesidad, dado que, al interactuar con sus compañeros en medio del juego, ya sean de cooperación, oposición o cooperación-oposición, facilitan la ejecución de actividades interválicas así como lo expresan López y Vicente (2018), señalando como los infantes diseñan en medio del juego o circuitos motores trabajos interválicos aeróbicos de alta intensidad de forma espontánea.

De acuerdo a lo anterior, es importante resaltar que la práctica de ejercicios de carácter cíclico bajo metodologías continuas resultan monótonas para los niños, de allí, que al prescribir programas de ejercicios, se debe optar por actividades placenteras y variadas (Hassink, 2010). En muchas de las propuestas de actividad física sistemática para niños, se plantean programas que no tienen en cuenta los principios pedagógicos, de planificación y metodología planteados a poblaciones infantiles, como es el caso del principio de promoción de la alegría en el entrenamiento, del doble esfuerzo, es decir, de entrenamiento y de formación, de la progresión y de la variedad entre otros (Martin, Nicolaus, Ostrowski, \& Rost, 2004).

Así mismo, cabe destacar que los resultados obtenidos en este estudio en las valoraciones del IMC, $\% \mathrm{GC}$ y VO $\mathrm{VP}_{2 \text { ico, }}$, demuestran cuan viable es implementar el HIIT como método de entrenamiento de la resistencia, en programas que tienen como finalidad disminuir el sobrepeso u obesidad y la prevención de enfermedades cardiovasculares, tal como lo afirman Bond et al. (2015) al señalar que este tipo de entrenamiento está asociado directamente a la mejora de la composición corporal y el componente cardiorrespiratorio.

Esta propuesta metodológica, basada en el HIIT, permite 
adaptaciones fisiológicas que favorecen la biogénesis mitocondrial, al respecto MacInnis y Gibala (2017) señalan cómo el incremento del número de mitocondrias facilita la oxidación de grasas en el organismo, además de estimular el metabolismo basal, aumentando de esta forma, la utilización de ácidos grasos como substrato energético en estado de reposo luego de la práctica del HIIT. Este proceso es conocido como exceso de consumo de oxígeno post ejercicio (EPOC) (Bahr \& Sejersted, 1991), al cual se le atribuye, que en etapa de recuperación se asocie al aclaramiento de lactato, incremento del funcionamiento pulmonar y cardiaco, elevación de la temperatura corporal, efectos de catecolaminas y resíntesis de glucógeno (Boutcher, 2011).

Uno de los aspectos claves en la utilización del HIIT, está relacionado con la identificación de las variables de carga adecuadas y su efecto positivo en la salud (Boutcher, 2011); al respecto, en los diferentes estudios que utilizaron el HIIT, el manejo de cargas fue diferente, aunque el medio de entrenamiento utilizado en varios de los estudios fue el cicloergómetro, como ocurrió con las investigaciones de Viñuela et al. (2016) y el de Heydari et al. (2012); la implementación de estrategias pedagógicas y medios de entrenamiento que provoque mayor adherencia a la práctica de ejercicios, en especial, en poblaciones infantiles y juveniles, es precisamente lo que suscitó el planteamiento del modelo de intervención bajo la metodología de las diferentes variantes del HIIT, utilizando como medio los juegos motores y circuitos sociomotrices, que precisamente también fueron utilizados por Segovia y Gutiérrez (2020) que recurrieron en este caso al HIIT con un enfoque lúdico, encontrando que esta metodología además de mejorar la condición física, promueve una mayor participación del alumnado, a pesar de su percepción de alta intensidad.

En cuanto a la metodología del entrenamiento HIIT utilizada, los estudios seleccionaron alguna de las variantes del HIIT. Por ejemplo, en una de las investigaciones se alternaron secuencias de esfuerzo de alta intensidad de 8 segundos con intervalos de recuperación activa de 12 segundos hasta completar 20 minutos en cicloergómetro (Heydari, et al., 2012); en el HIIT propuesto por Galdames-Maliqueo et al. (2017) las secuencias de esfuerzo de alta intensidad se realizaban en un espacio de 20 metros con cambio de dirección, tomando como referencia 10 segundos, el cual va variando al incrementar la intensidad de esfuerzo por mayor exigencia en recorrido, al terminar la secuencia de esfuerzo de alta intensidad, los escolares descansan 20 segundos, para iniciar nuevamente.

Ahora, en la propuesta de HIIT de Camacho et al. (2016), las secuencias de esfuerzo de alta intensidad se limitaban a realizar de cuatro a seis series de sprints de 20 segundos con secuencias de recuperación de 20 a 60 segundos. Por otra parte, Viñuela et al. (2016) utilizaron el HIIT con secuencias de esfuerzo de alta intensidad de 30 segundos en cicloergómetro, con secuencias de recuperación activa de 4 minutos. Como se puede apreciar, en cada estudio se toma como referencia una sola metodología de las variantes del HIIT, que a diferencia de la propuesta realizada con la población infantil con sobrepeso u obesidad en Tuluá, propuso diversas variantes del HIIT por etapas, desde secuencias de esfuerzo de alta intensidad que duraron de 2 a 4 minutos hasta esfuerzos de 3 a 7 segundos. De acuerdo a lo anterior, se infiere que el aumento del $\mathrm{VO}_{2 \text { ico }}$ y la disminución IMC y $\% \mathrm{GC}$ de la población de niñas con sobrepeso u obesidad, en especial estos dos últimos, evidencian la efectividad del método de entrenamiento HIIT, utilizando en este caso, juegos motores y circuitos sociomotrices, solo que en la investigación realizada en Tuluá con las niñas se recurrieron a actividades motoras de carácter acíclico e interválico de diferente duración en cada una de las fases y el uso del juego como medio de entrenamiento, para así lograr una mayor motivación en la población intervenida.

Es importante resaltar, que en otras investigaciones donde no utilizaron la metodología HIIT, igualmente se presentaron efectos positivos en los componentes antropométricos, como sucedió en el estudio con adolescentes con exceso de peso que utilizó el ejercicio aeróbico de carácter continuo a través de banda sin fin (Vargas-Ortiz, et al., 2014) ó, el realizado con la población escolar con obesidad que utilizó el ejercicio aeróbico mediante actividades polideportivas combinado ejercicios de fuerza y flexibilidad (García-Hermoso, Escalante, Domínguez, \& Saavedra, 2013).

Sin embargo, cabe señalar que estos métodos de entrenamiento por su naturaleza, requieren de una mayor cantidad de tiempo de trabajo en sus respectivas sesiones, lo que puede convertirse en una limitante para los escolares (Stutts, 2002). De allí que, el entrenamiento HIIT utilizando circuitos y juegos motores de interacción (cooperación-oposición) se convierte en una alternativa interesante en la estructuración de programas de actividad física con enfoque salud, por su particularidad de presentar adaptaciones fisiológicas similares al entrenamiento aeróbico continuo con tiempos de trabajo mucho más cortos, como lo han señalado diferentes autores a lo largo del presente estudio (Wisløff, Ellingsen, \& Kemi, 2009).

\section{Conclusiones}

El programa de entrenamiento HIIT que utilizó como medios los juegos motores y circuitos sociomotrices, presentó efectos estadísticamente significativos. Primero en la disminución del índice de masa corporal y porcentaje de grasa, y segundo, en el incremento del consumo de oxigeno pico de las niñas de 10 y 11 años con sobrepeso u obesidad de la sede Marino Dávalos de la Institución Educativa Moderna de Tuluá.

\section{Nota aclaratoria}

Los autores de este artículo expresan no tener ningún conflicto de interés que sea relevante para el contenido de este manuscrito.

\section{Referencias}

Alba,A. (2010). Test funcionales, cineantropometría y prescripción del entrenamiento en el deporte y la actividad física (Tercera ed.). Armenia: Kinesis.

Álvarez, C.E.,Herrera, M.F., Herrera, E., Villalobos, G., \&Araya, G. (2020). Sobrepeso, obesidad, niveles de actividad fisica y autoestima de la niñez centroamericana: un análisis compa- 
rativo entre países. Retos, 37(37), 238-243. Recuperado de https://recyt.fecyt.es/index.php/retos/article/view/71680

Bahr, R., \& Sejersted, O. M. (1991). Effect of intensity of exercise on excess postexercise $0_{2}$ consumption. Metabolism, $40(8)$, 836-841.doi:https://doi.org/10.1016/0026-0495(91)90012-L

Bailey, R. C., Olson, J., Peper, S., Porszasz, J., Barstow, T. J., \& Cooper, D. M. (1995). The level and tempo of children's physical activities: an observational study. Medicine and science in sports and exercise, 27(7), 1033-1041. doi: https:/ /doi.org/10.1249/00005768-199507000-00012

Bond, B., Cockcroft, E., Williams, C., Harris, S., Gates, P., Jackman, S.,... Barker,A. (2015). Two weeks of high-intensity interval training improves novel but not traditional cardiovascular disease risk factors in adolescents. American journal of physiology. Heart and circulatory physiology, 309(6), 10391047.doi: https://doi.org/10.1152/ajpheart.00360.2015

Boutcher, S. (2011). High-Intensity Intermittent Exercise and Fat Loss. Journal of obesity, 1254-1259. doi: https://doi.org/ 10.1155/2011/868305

Buchheit, M., \& Laursen, P. (2013). High-Intensity Interval Training, Solutions to the Programming Puzzle: Part I: cardiopulmonary emphasis. Sports medicine, 43(5), 313-338. doi:https://doi.org/10.1007/s40279-013-0029-x

Camacho,A., Brazo, J., Camacho, M., Marcos, M., Timón, R., \& Olcina, G. (2016). Efecto de un protocolo de entrenamiento interválico de alta intensidad sobre masa grasa corporal en adolescentes. Revista Española de Salud Pública, 90, 1-9. Recuperado de http://scielo.isciii.es/pdf/resp/v90/1135-5727resp-90-e40024.pdf

Crisp, N.A., Fournier,P.A., Licari, M. K., Braham, R., \& Guelfi, K. J.(2012). Adding sprints to continuous exercise at the intensity that maximises fat oxidation: Implications for acute energy balance and enjoyment. Metabolism: clinical and experimental, 61(9), 1280-1288. doi: https://doi.org/10.1016/ j.metabol.2012.02.009

FAO \& OPS. (19 deEnero de 2017). FAO/OPS: sobrepeso afecta a casi la mitad de la población de todos los países de América Latina y el Caribe salvo por Haití. Oficina Regional de laFAO para América Latina y el Caribe: Recuperado de http:// www.fao.org/americas/noticias/ver/es/c/463396/

Galdames-Maliqueo, S., Huerta-Ojeda, Á., Chirosa-Ríos, L., Cáceres-Serrano, P., \& Reyes-Amigo, T.(2017). Efecto de un método de Entrenamiento Intervalado de Alta Intensidad sobre el Consumo Máximo de Oxígeno en Escolares Chilenos. UniversidadySalud,19(3),359-365. doi: http://dx.doi.org/ 10.22267/rus. 171903.98

García-Hermoso,A., Escalante, Y., Domínguez,A.M., \& Saavedra, J.M. (2013). Efectos de un programa de ejercicio físico durante tres años en niños obesos. Retos, (23), 10-13. Recuperado dehttps://recyt.fecyt.es/index.php/retos/article/view/34559

Gibala, M., Little, J., MacDonald, M., \& Hawley, J. (2012). Physiological adaptations to low-volume, high-intensity interval training in health and disease. The Journal of physiology,590(5), 1077-1084. doi: https://doi.org/10.1113/ jphysiol.2011.224725

Hassink, S. (2010). Obesidad Infantil. prevención, intervenciones y tratamiento en atención primaria. Madrid: Médica Panamericana.

Hebestreit, H., Mimuray, K.-I., \& Bar-Or, O. (1993). Recovery of muscle power after high-intensity. Journal of applied physiology, 74(6), 2875-2880. doi: https://doi.org/10.1152/ jappl.1993.74.6.2875

Heydari, M., Freund, J., \& Boutcher, S. (2012). TheEffect ofHighIntensity Intermittent Exercise on Body Composition of Overweight Young Males. Journal of Obesity, 34(3), 1-8. doi: https://doi.org/10.1155/2012/480467

Imperial College de Londres \& OMS. (11 de Octubre de 2017). La obesidad entre los niños y los adolescentes se ha multiplicado por 10 en los cuatro últimos decenios. Recuperado de: http://www.who.int/es/news-room/detail/11-10-2017-tenfoldincrease-in-childhood-and-adolescent-obesity-in-fourdecades-new-study-by-imperial-college-london-and-who

Katch, V.L., McArdle,W.D., \& Katch, F. L. (2014). Fisiología del ejercicio: fundamentos (Cuarta ed.). Madrid: Médica Panamericana.

Laursen, P., \& Buchheit, M. (2019). Science and application of high-intensity interval training. Champaign: Human Kinetics.

López, J., \& Vicente, D. (2018). HIIT, Entrenamiento interválico de alta intensidad, bases fisiológicas y aplicaciones prácticas. (J.López, Ed.) Madrid:Autor editor.

MacInnis, M. J., \& Gibala, M. J.(2017). Physiological adaptations to interval training and the role of exercise intensity. The Journal of physiology, 595(9), 2915-2930. doi: https://doi.org/ 10.1113/JP273196

Martin, D., Nicolaus, J., Ostrowski, C., \& Rost, K. (2004). Metodología general del entrenamiento infantil y juvenil. Barcelona: Paidotribo.

Ministerio de Salud \& Protección Social. (21 de Noviembre de 2017). Gobierno presenta Encuesta Nacional de Situación Nutricional de Colombia(ENSIN) 2015. Recuperado de https:/ /www.minsalud.gov.co/Paginas/Gobierno-presenta-Encuesta-Nacional-de-Situaci\%C3\%B3n-Nutricional-de-ColombiaENSIN-2015.aspx

Ministerio de Salud y Protección Social. (14 de Junio de 2016). Resolución No. 2465 de 2016. Recuperado de https:// www.minsalud.gov.co/Normatividad_Nuevo/Forms/ DispForm.aspx?ID=4909

Segovia, Y., \& Gutiérrez, D. (2020). Esfuerzo, Implicacióny Condición Física percibida en un Programa HIIT en Educación Física. Modelo Educación Deportiva vs Metodología Tradicional. Retos, 38(38), 151-158. Recuperado de https:// recyt.fecyt.es/index.php/retos/article/view/73686

Stutts, W. (2002). Physical activity determinants in adults. Perceived benefits, barriers, and self efficacy. $A A O H N$ Journal, 50(11), 499-507. doi: https://doi.org/10.1177/ 216507990205001106

Vargas-Ortiz, K., Macías-Cervantes, M. H., Díaz-Cisnero, F. J., \& Pérez-Vázquez, V. (2014). El entrenamiento aeróbico de 12 semanas reduce los factores de riesgo cardiovascular en adolescentes con exceso de peso. Gaceta Médica de México, 150(Supp 1), 120-124. Recuperado de https:// www.medigraphic.com/pdfs/gaceta/gm-2014/gms141q.pdf Viñuela, M., Vera, A., Colomer, D., Márquez, G., \& Romero, S. (2016). Efecto de 12 sesiones de un entrenamiento interválico de alta intensidad sobre la composición la corporal en adultos jóvenes. Nutrición Hospitalaria, 33(3), 637-643. doi: http://dx.doi.org/10.20960/nh.272

Wisløf,,U.,Ellingsen, Ø.,\& Kemi, O.(2009). High-intensity interval training to maximize cardiac benefits of exercise training? Exercise and Sport Sciences Reviews, 37(3), 139-146. doi: https://doi.org/10.1097/JES.0b013e3181aa65fc 